\title{
Proof for Eq. (15)
}

Here, we prove that a mutant on the cycle with dB updating has lower fixation probability than in the well-mixed population for general $N$. We have to show that $\phi_{d B}^{M}-\phi_{d B}^{\circ}>0$ for $r>0, r \neq 1$. Under $\mathrm{dB}$ updating, the fixation probability in the well-mixed population is given by

$$
\phi_{d B}^{M}=\frac{N-1}{N} \frac{1-\frac{1}{r}}{1-\frac{1}{r^{N-1}}} .
$$

On the cycle, the respective fixation probability is given by (Eq. (5.3) in [1])

$$
\phi_{d B}^{\circ}=\frac{2(r-1)}{3 r-1+(r-3) r^{2-N}} .
$$

Then the difference is given by

$\phi_{d B}^{M}-\phi_{d B}^{\circ}=\frac{(r-1) r^{N-2}}{N} \cdot \frac{(N-3) r^{N+1}-(N-1) r^{N}+(N-1) r^{3}-(N-3) r^{2}}{\left(r^{N-1}-1\right)\left(3 r^{N+1}-r^{N}+r^{3}-3 r^{2}\right)}$

This expression can be written in the form of

$$
\frac{(r-1)^{2} r^{N-2}}{N} \cdot \frac{\sum_{k=0}^{N-4} r^{k}\left((N-2)(k+1)-(k+1)^{2}\right)}{\left(\sum_{k=0}^{N-2} r^{k}\right)\left(3+\sum_{k=1}^{N-3} 2 r^{k}+3 r^{N-2}\right)} .
$$

As Eq. (4) contains only positive coefficients in $r$, the difference is always positive for $r>0$ and $r \neq 1$. For $r=1$, it is zero. Thus Eq. (15) is fulfilled for all $r>0, r \neq 1$.

\section{References}

[1] Kaveh K, Komarova NL, Kohandel M. The duality of spatial deathbirth and birth-death processes and limitations of the isothermal theorem. Journal of the Royal Society Open Science. 2015;2(140465). 